\title{
Post-Starburst Populations Near and Far - The Potential of Near-IR Spectroscopy
}

\author{
Ariane Lançon ${ }^{1}$ and Mustapha Mouhcine ${ }^{1}$ \\ Observatoire de Strasbourg (UMR 7550), 11 rue de l'Université, F-67000 \\ Strasbourg, France
}

\begin{abstract}
The efficient use of near-IR data in studies of external stellar populations depends on our ability to recognize the nature of the predominant sources of light, and to interprete these findings in terms of age and metallicity. Here we focus on elderly post-starburst populations, with ages of $10^{8}-10^{9} \mathrm{yrs}$. New models confirm that they are indeed expected to display specific spectral signatures in the near-IR, due to variable $\mathrm{M}$ stars of the asymptotic giant branch and to carbon stars. The signatures depend on age and metallicity. We summarize the status of current quantitative predictions and emphasize the importance of an empirical calibration of the spectral synthesis models.
\end{abstract}

\section{Overview}

Near-IR data has been used very often to confirm the presence of an "evolved stellar component" in starburst galaxies. The need to study stellar populations deeply embedded in dust is also frequently invoked to justify near-IR observations. But what exactly are these cool stellar populations seen between 1 and $3 \mu \mathrm{m}$ ? Population synthesis models tell us that red supergiants are the main sources $10^{7}-10^{8}$ yrs after a star formation episode; during the following $10^{9} \mathrm{yrs}$, stars of the upper, thermally pulsing asymptotic giant branch (TP-AGB) become predominant; and finally giants of the early AGB (E-AGB) and of the first red giant branch (RGB) outweigh other populations. The efficient use of near-IR data depends on our ability to distinguish these categories from each other and to interprete them in terms of age and metallicity.

Very strong $\mathrm{CO}$ absorption $(2.3 \mu \mathrm{m})$ is a clear signature of a red supergiant population ([1 initiated the systematic exploration of CO in cool stars; [19] analysed early observations in starbursts; 9 discussed the diagnostic power of the feature and its limitations). But the dependence of the red supergiant population on metallicity is strong [14 and not reproduced by stellar evolution models unless ad hoc corrections are invoked ([12], [17]). Therefore the threshold above which the $\mathrm{CO}$ absorption becomes an unambiguous signature of a supergiant population is uncertain. On the other hand, it has recently been claimed that high quality, high spectral resolution near-IR observations allow for relative age-dating of young post-starburst populations to within $2-3 \mathrm{Myr}$, at least at solar metallicity (Gilbert, this workshop). 
The focus of our work is on somewhat older post-starburst populations, such as we are likely to find in evolved mergers or " $\mathrm{E}+\mathrm{A}$ " galaxies, i.e. after the most obvious signs of a recent starburst have faded away. Between $10^{8}$ and $10^{9} \mathrm{yrs}$ of age, of the order of $50 \%$ of the $\mathrm{K}$ band light of a coeval population originates from TP-AGB stars. Large stellar samples show that essentially all the TP-AGB stars are long period variables [21]. Spectral libraries as well as model atmospheres demonstrate that this pulsation results in deeper $\mathrm{H}_{2} \mathrm{O}$ absorption bands than seen in E-AGB or RGB stars. This provides us with a means of identifying strong contributions of oxygen-rich (e.g. M type) TPAGB stars. In addition, the convective dredge-up of carbon-rich material from the stellar core into the envelope progressively turns some TP-AGB stars into carbon stars. Those again have unmistakable spectral signatures in the nearIR. Finally, TP-AGB stars may become dust enshrouded mid-IR sources, and it has been suggested that the presence of these modifies the location of stellar populations in near-IR versus mid-IR two-colour diagrams [3]. All the mentioned processes depend sensitively on the initial stellar mass (and thus on post-starburst age) as well as on metallicity. This was the motivation for our work. We summarize its status below.

\section{Model construction and predictions}

Our population synthesis models are based on the code structure of PÉGASE [5], the stellar tracks of the "Padova Group" ([2], [4] ) up to the end of the EAGB, and the spectral library of Lejeune et al. 13]. We have completed this set of inputs with synthetic evolutionary tracks for the TP-AGB that incorporate the effects of mass loss, dredge-up and envelope burning of dredged-up materials ([15], [16]); we also use a new library of cool stellar spectra 11.

Various global properties can be determined without invoking spectral libraries, on the basis of the TP-AGB tracks alone 16]. The values given here are based on our currently prefered internal model parameters (e.g. mass loss prescription, convective mixing length, dredge-up efficiency), which have been tested against statistical properties in nearby stellar populations (see below). The stars with the longest TP-AGB durations (and thus the largest luminous energy output, integrated along the TP-AGB) are found to have initial masses $\left(M_{i}\right)$ between 2 and $2.5 \mathrm{M}_{\odot}$ (i.e. ages of 0.7 to $1.1 \mathrm{Gyr}$ ); stars with $M_{i}$ in the $1.7-3 \mathrm{M}_{\odot}$ range last $\sim 10^{6} \mathrm{yrs}$ or more in that phase. Mass loss has the strongest direct influence on these durations, and itself depends on various other model parameters through the instantaneous masses, luminosities and radii. As the TP-AGB stars are the coolest luminous stars present, the duration of that phase essentially controls the (V-K) colour of the stellar population [6]. The fraction of the TP-AGB duration spent as a carbon star is found to be maximal for objects with initial masses between 2.5 and $3 \mathrm{M}_{\odot}$ (i.e. ages of 0.4 to $0.8 \mathrm{Gyr}$ ), where it typically amounts $45 \%$ at solar metallicity and about $80 \%$ at the metallicity of the Large Magellanic 
Cloud (Fig. 1). Due to strong mass loss, stars above $M_{i}=3.5 \mathrm{M}_{\odot}$ have relatively short TP-AGB durations $\left(<5 \times 10^{5} \mathrm{yrs}\right)$ and are embedded in dust for a significant fraction of this time. This reduces the predicted contribution of TP-AGB stars to the light at ages of $0.1-0.4 \mathrm{Gyr}$, when compared to our earlier models [10].
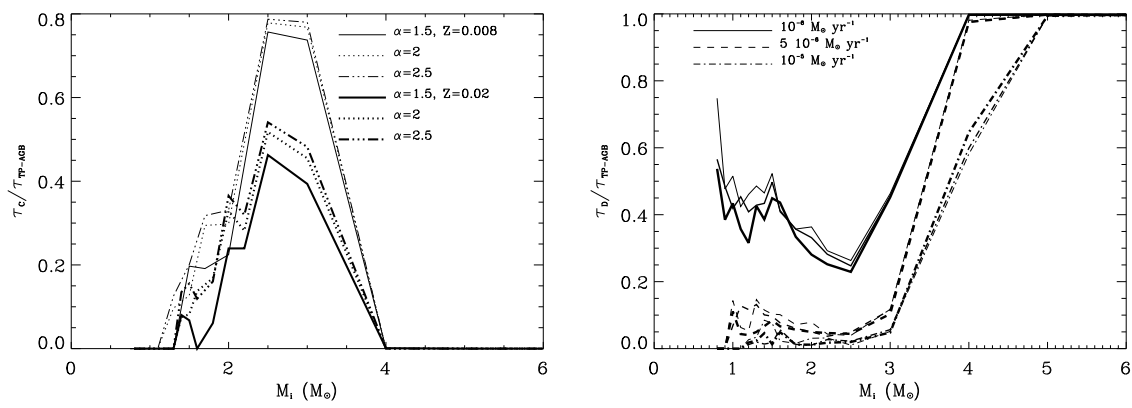

Fig. 1. Left: Fraction of the TP-AGB duration spent as a C-star, as a function of initial stellar mass. Two metallicities and various values of the mixing length parameter are shown. Right: Fraction of the TP-AGB duration spent as a star with mass loss above $10^{-6}, 5 \times 10^{-6}$ and $10^{-5} \mathrm{M}_{\odot} / \mathrm{yr}$, at solar metallicity.

As mentioned, our trust in the model prediction is based on a variety of tests. For instance, the new evolutionary models for the TP-AGB successfully reproduce the empirical initial to final mass relation for stars in the solar neighbourhood. The predicted contributions of C-stars to the near-IR light of intermediate age populations are consistent with those observed in the Magellanic Clouds (e.g. [18]). One of us (M.M.) has coupled the TP-AGB models with chemical evolution models for galaxies (including the yields of intermediate mass stars as given by the new tracks). The tight relations observed in the Local Group galaxies between metallicity and the mean luminosity of C-stars, or between metallicity and the C-star/M-star number ratio can be reproduced very naturally. These results will be discussed in detail elsewhere.

Stellar libraries are required for the prediction of near-IR colours or spectral features. Based on the extremely diverse AGB star spectra of [11] $(\lambda / \delta \lambda \simeq$ 1100 between 1 and $2.5 \mu \mathrm{m}$ ), we have constructed a regular sequence of averages that can be more conveniently coupled with population synthesis models [8]. As a result, we predict that the signatures of upper AGB stars are seen in the integrated light of a stellar populations between $\sim 0.2$ and $\sim 1.2 \mathrm{Gyr}$ after a starburst (16], [10]). The main signatures are extremely broad, and best detected with low resolution data with very wide spectral coverage (the $\mathrm{K}$ band spectrum alone is not sufficient). As the features reach accross telluric absorption bands, observations at good IR sites are essential. The features are due to $\mathrm{H}_{2} \mathrm{O}$ if the TP-AGB stars are O-rich, to $\mathrm{C}_{2}$ and $\mathrm{CN}$ if they are 
C-rich. Narrow band indices can be defined that detect TP-AGB stars independently of their chemical nature, and others that then separate the two classes 10 . Photometry with an accuracy better than $5 \%$ must be aimed for. We are exploring the feasibility of surveys with the available filters on the NICMOS camera onboard the Hubble Space Telescope.

\section{The need for empirical calibration}

Once TP-AGB evolutionary tracks have been constructed and tested, only a part of the work is done: associating a spectrum with a given point of the theoretical HR diagram is difficult, as illustrated in Fig. 2. Individual stars vary significantly in luminosity and colour temperature. While optical colours and temperature indicators such as $(\mathrm{V}-\mathrm{K})$ correlate well, the near-IR properties (colours, strength of the $\mathrm{H}_{2} \mathrm{O}$ or $\mathrm{CO}$ bands) are very dispersed at a given colour temperature. In addition, no model atmospheres are available to reliably relate an instantaneous colour temperature to the effective temperature of the simple static stars on which evolutionary tracks are based. The regularity of the spectral sequence obtained by [8] gives some confidence in the evolution of the spectra with temperature; but only empirical calibrations, based on stellar populations with ages and metallicities known from optical observations, can constrain absolute relationships.
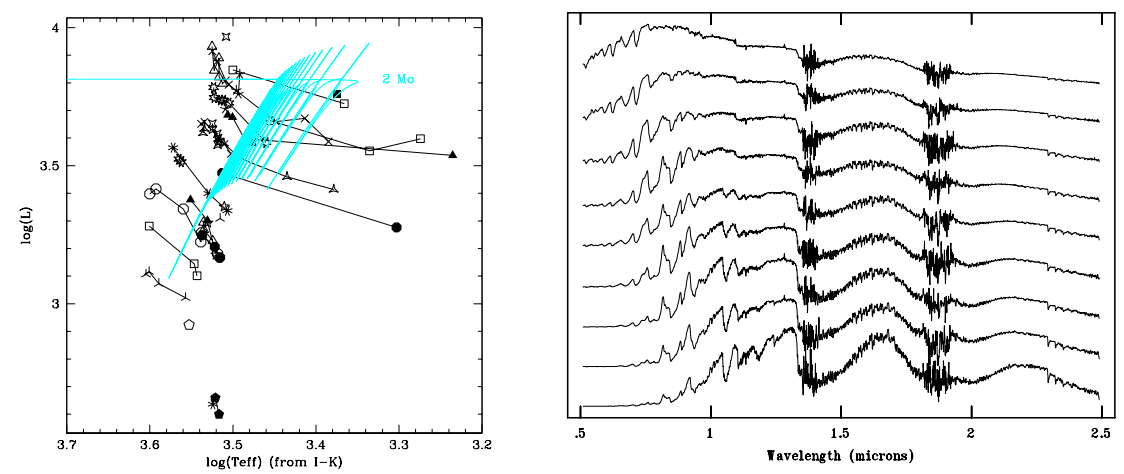

Fig. 2. Left: Estimated location of individual spectra of [1] in the HR diagram. Multiple observations of individual stars are connected with lines. An illustrative TP-AGB track for a $2 \mathrm{M}_{\odot}$ star is also shown. Right: Empirical sequence of average O-rich TP-AGB star spectra [8].

Despite the more numerous near-IR instruments now available on large telescopes, appropriate targets for the model calibration remain hard to find. The LMC and M33 clusters, of which several have adequate ages, tend to be too small: they contain a handful of TP-AGB stars and their integrated 
properties are affected by the stochastic nature of this subpopulation. In [11, it was shown that stellar populations with total masses of $\sim 10^{5} \mathrm{M}_{\odot}$ are needed to obtain significant constraints on the TP-AGB models. Massive stellar clusters around merging galaxies are well suited, but only a few are bright enough for near-IR spectroscopy. We have observed the $500 \mathrm{Myr}$ old cluster W3 in NGC 7252 with SOFI (NTT, ESO), between 1 and $2.4 \mu \mathrm{m}$. Illustrative adjustments with models are shown in Fig. 3 . The figures illustrate the need for broad wavelength coverage and excellent relative calibrations of the various atmospheric windows (with SOFI the overlap between grisms ensures this). Our preliminary conclusions are the following. The data reject models that have few $\mathrm{C}$-stars and use a cool effective temperature scale for the TP-AGB spectra: the water bands in those are too strong. This suggests that the metallicity is not more than solar and that the coolest of the Mira temperature scales in the literature is inappropriate for our purpose. Good fits to the spectral shape are obtained at solar and LMC metallicities, with extinction values compatible with optical estimates 20. More calibration points will be required, but this first test already restricts the allowed range of parameters and gives us confidence in the new tool.
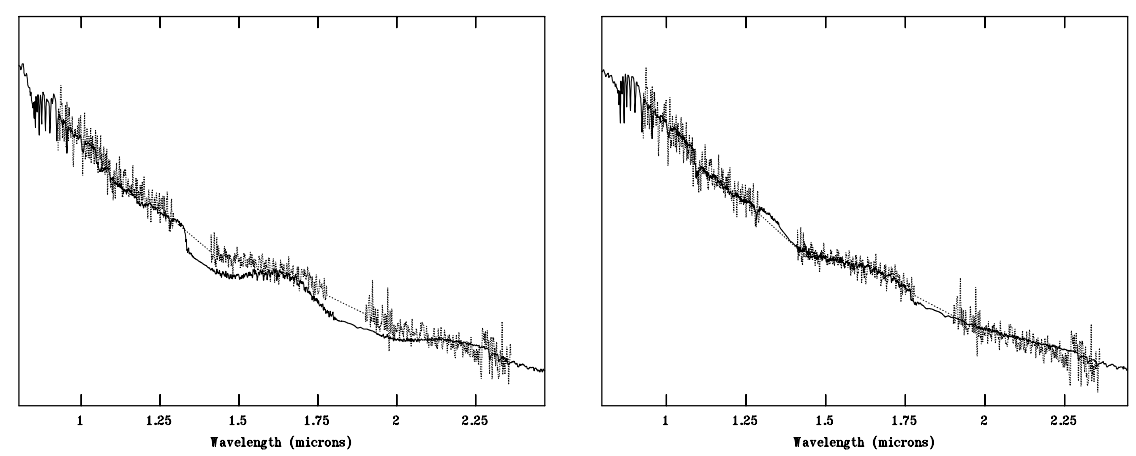

Fig. 3. The observed spectrum of $\mathrm{W} 3$ and two illustrative models. Left: solar metallicity, formation of C-stars switched off, cool TP-AGB temperature scale. This model is ruled out. Right: LMC metallicity, formation of C-stars allowed. This model matches the data well.

\section{Conclusion}

Based on new synthetic evolution models for the TP-AGB and on a purpose designed library of stellar spectra, we have shown that near-IR spectra of post-starburst populations indeed carry information about post-starburst age and metallicity. Elderly post-starbursts with ages of $10^{8}$ to $10^{9} \mathrm{yrs}$ can be distinguished from younger or older ones, using the specific spectral signatures 
of O-rich and C-rich TP-AGB stars. The chemical nature of the predominant TP-AGB stars is a metallicity indicator. The population of dust-obscured TP-AGB stars depends both on age and metallicity. We have pointed out that the relevant spectral features can be detected at low spectral resolution as long as a broad continuous spectral coverage is obtained. High quality narrow band photometry can also be used. The quantitative prediction of the strength of the near-IR features must be based on empirical calibrations. We have described the current status of these.

Applications of this work include studies of the duration of star formation episodes in starburst galaxies or mergers, of the survival timescale of young starburst clusters in the galaxy environment, and of the nature of " $\mathrm{E}+\mathrm{A}$ " galaxies. First observations of "E+A" galaxies will be available to us soon.

\section{References}

1. J.R. Baldwin, J.A. Frogel, S.E. Persson: ApJ 184, 427 (1973)

2. A. Bressan, F. Fagotto, G. Bertelli, C. Chiosi: A\&AS 100, 647 (1993)

3. A. Bressan, G.L. Granato, L. Silva: A\&A 332, 135 (1998)

4. F. Fagotto, A. Bressan, G. Bertelli, C. Chiosi: A\&AS 105, 29 (1994)

5. M. Fioc, B. Rocca-Volmerange: A\&A ...

6. L. Girardi, G. Bertelli: MNRAS 300, 533 (1998)

7. A. Lançon, C. Boily (eds.): Massive Stellar Clusters, ASP Conf. Ser. 211 (ASP, San Francisco 2000)

8. A. Lançon, M. Mouhcine. In preparation (2001)

9. A. Lançon, B. Rocca-Volmerange: New Astron. 1, 215 (1996)

10. A. Lançon, M. Mouhcine, M. Fioc, D. Silva: A\&A 344, L21 (1999)

11. A. Lançon, P.G. Wood: A\&AS 146, 217 (2000)

12. N. Langer, A. Maeder: A\&A 295, 685 (1995)

13. T. Lejeune, F. Cuisinier, R. Buser: A\&AS 125, 229 (1997)

14. P. Massey: AJ 501, 153 (1998)

15. M. Mouhcine, A. Lançon: In [7], p. 144 (2000)

16. M. Mouhcine, A. Lançon. In preparation (2001)

17. L. Origlia, J.D. Goldader, C. Leitherer, D. Schaerer, E. Oliva: ApJ 514, 96 (1999)

18. S.E. Persson, M. Aaronson, J.G. Cohen, J.A. Frogel, K. Matthews: ApJ 266, 105 (1983)

19. G.H. Rieke, M.J. Lebofsky, R.I. Thompson, F.J. Low, A.T. Tokunaga: ApJ 238, 24 (1980)

20. F. Schweizer, P. Seitzer: AJ 116, 2206 (1998)

21. P.G. Wood: In AGB stars, IAU Symp. 192. 